\title{
Number OF Eigenvalues OF NON-SELF-ADJOINT SCHRÖDINGER Operators with Dilation Analytic Complex Potentials
}

\author{
Norihiro Someyama *
}

\begin{abstract}
In the present paper, we give Lieb-Thirring type inequalities for all isolated eigenvalues of $d$-dimensional non-self-adjoint Schrödinger operators with complex-valued and dilation analytic potentials. In order to derive them, we prove that isolated eigenvalues and their multiplicities are invariant under complex dilation.
\end{abstract}

Keywords: non-self-adjoint Schrödinger operator, Lieb-Thirring inequality, complex potential, dilation analytic potential, isolated eigenvalue

\section{Introduction}

In the present paper, we consider Schrödinger operators on $\mathbb{R}^{d}$,

$$
H:=H_{0}+V, \quad H_{0}:=-\Delta=-\sum_{j=1}^{d} \frac{\partial^{2}}{\partial x_{j}^{2}}
$$

with complex potentials $V$ which are dilation analytic (see Assumption 2.1 for definition), where the domain $\mathcal{D}\left(H_{0}\right)$ of $H_{0}$ is the second order Sobolev space in $\mathbb{R}^{d}, H^{2}\left(\mathbb{R}^{d}\right)$. We denote the real and the imaginary parts of $z \in \mathbb{C}$ by $\Re z$ and $\Im z$ respectively. We prove that the sum of moments of isolated

${ }^{*}$ Department of Mathematics, Gakushuin University, 1-5-1 Mejiro, Toshima-ku, Tokyo 171-8588, Japan / e-mail: philomatics@outlook.jp 
eigenvalues of $H$ in the half-plane can be estimated by the Lieb-Thirring type inequalities

$$
\sum_{\lambda \in \sigma_{\mathrm{d}}(H) \cap\left[\mathbb{C}^{ \pm} \cup(-\infty, 0)\right]}|\lambda|^{\gamma} \leq C_{\gamma, d} \int_{\mathbb{R}^{d}}\left|V_{ \pm \pi \mathrm{i} / 4}(x)\right|^{\gamma+d / 2} \mathrm{~d} x
$$

for $d, \gamma \geq 1$, where $\mathbb{C}^{ \pm}:=\{z \in \mathbb{C}: \pm \Im z>0\}$ and $V_{\theta}(x):=V\left(\mathrm{e}^{\theta} x\right)$. Here and hereafter, the formulas which contain \pm represent two formulas, one for the upper sign and the other for the lower sign. The space of $L^{p}$-functions from the space $X$ to the space $Y$ will be denoted by $L^{p}(X ; Y)$, in particular we denote $L^{p}(X ; X)=L^{p}(X)$.

In addition, many researches on the number of eigenvalues of Schrödinger operators with complex potentials have been done. For instance, Frank, Laptev and Safronov([13]) recently derived the following result for the number $N$ of isolated eigenvalues of the odd dimensional Schrödinger operator $H$ with the complex potential which decays exponentially fast:

$$
N \leq \frac{C_{d}}{\varepsilon^{2}}\left(\int_{\mathbb{R}^{d}} \mathrm{e}^{\varepsilon|x|}|V(x)|^{(d+1) / 2} \mathrm{~d} x\right)^{2}
$$

for any $\varepsilon>0$, where $C_{d}$ is a constant depending only on $d=3,5,7, \ldots$. We also give an inequality for the number of all isolated eigenvalues of any dimensional Schrödinger operator $H$ with the special dilation analytic complex potential as a corollary of (1.1).

\subsection{Lieb-Thirring Inequalities for Real Potentials}

We recall the standard Lieb-Thirring inequality. We consider the self-adjoint Schrödinger operator $H=H_{0}+V$ in $L^{2}\left(\mathbb{R}^{d}\right)$ defined by the closure of the quadratic form

$$
q_{H}(u):=\int_{\mathbb{R}^{d}}|\nabla u(x)|^{2} \mathrm{~d} x+\int_{\mathbb{R}^{d}} V(x)|u(x)|^{2} \mathrm{~d} x, \quad u \in C_{0}^{\infty}\left(\mathbb{R}^{d}\right)
$$

where $\nabla:=\left(\partial_{x_{j}}\right)_{1 \leq j \leq d}$ with the derivative $\partial_{x_{j}}$ in the sense of distibutions. If $V \in L^{\gamma+d / 2}\left(\mathbb{R}^{d} ; \mathbb{R}\right)$ and

$$
\lambda_{1} \leq \lambda_{2} \leq \cdots \leq \lambda_{n} \leq \cdots<0
$$

are negative eigenvalues of $H$, the standard Lieb-Thirring inequality:

$$
\sum_{n=1}^{\infty}\left|\lambda_{n}\right|^{\gamma} \leq L_{\gamma, d} \int_{\mathbb{R}^{d}} V_{-}(x)^{\gamma+d / 2} \mathrm{~d} x, \quad V_{ \pm}:=\max \{ \pm V, 0\}
$$


is well known(e.g., [3, [18], [19], [21], 22] and [23]), here $L_{\gamma, d}$ is the sharp constant and $\gamma$ satisfies

$$
\left.\begin{array}{l}
\gamma \geq \frac{1}{2}, \quad d=1 \\
\gamma>0, \quad d=2 \\
\gamma \geq 0, \quad d \geq 3
\end{array}\right\}
$$

It is important that $L_{\gamma, d}$ do not depend on $V$. If $\gamma=0$, the left-hand side of (1.3) is the number of negative eigenvalues (1.2) of $H$. For the constant $L_{\gamma, d}$, it is well known that

$$
L_{\gamma, d}^{\mathrm{cl}} \leq L_{\gamma, d}
$$

for all $\gamma \geq 0$ and $d \geq 1$, where $L_{\gamma, d}^{\mathrm{cl}}$ is the classical constant:

$$
L_{\gamma, d}^{\mathrm{cl}}:=(2 \pi)^{-d} \int_{\mathbb{R}^{d}}(|\xi|-1)_{-}^{\gamma} \mathrm{d} \xi=\frac{\Gamma(\gamma+1)}{2^{d} \pi^{d / 2} \Gamma(\gamma+d / 2+1)} .
$$

In fact, it is proven that

$$
L_{\gamma, d}=L_{\gamma, d}^{\mathrm{cl}}
$$

for all $d \geq 1$ if $\gamma \geq 3 / 2$ (cf. [23],[3],[19]), and

$$
L_{\gamma, d} \leq \frac{\pi}{\sqrt{3}} L_{\gamma, d}^{\mathrm{cl}}=(1.814 \cdots) \times L_{\gamma, d}^{\mathrm{cl}}
$$

for all $d, \gamma \geq 1([9])$. Frank, Hundertmark, Jex and Nam([1] $)$ however improved (1.6) to

$$
L_{\gamma, d} \leq 1.456 \times L_{\gamma, d}^{\mathrm{cl}}
$$

if $\gamma=1$. On the other hand, Helffer and Robert([15]) proved that

$$
L_{\gamma, d}^{\mathrm{cl}}<L_{\gamma, d}
$$

if

$$
\left.\begin{array}{ll}
\frac{1}{2} \leq \gamma<\frac{3}{2}, & d=1 \\
\gamma<1, & d \geq 2
\end{array}\right\} .
$$

For further information about $L_{\gamma, d}$ and $L_{\gamma, d}^{\mathrm{cl}}$, see Remarks of Theorem 12.4 of [21].

Lieb-Thirring inequalities are key ingredients in the proof of the stability of matter by Lieb and Thirring(e.g., [22]), and is used for obtaining the efficient lower bound for the energies of fermions. 


\subsection{Lieb-Thirring Inequalities for Complex Potentials}

The Lieb-Thirring inequality (1.3) has recently been extended to Schrödinger operators with complex potentials $V \in L^{\gamma+d / 2}\left(\mathbb{R}^{d} ; \mathbb{C}\right)$ for $d, \gamma \geq 1$.

We suppose that $V$ is a complex-valued potential which is $H_{0}$-compact. Then

$$
H=H_{0}+V, \quad \mathcal{D}(H)=\mathcal{D}\left(H_{0}\right)
$$

is quasi-maximal accretive([16]), and the spectrum $\sigma(H)$ of $H$ consists of the essential spectrum $\sigma_{\text {ess }}(H)=[0, \infty)$ and the discrete spectrum $\sigma_{\mathrm{d}}(H)$ which consists of isolated eigenvalues of $H$ of finite algebraic multiplicities:

$$
m_{\lambda}:=\sup _{k \in \mathbb{N}}\left(\operatorname{dim} \operatorname{ker}\left[(H-\lambda)^{k}\right]\right) .
$$

This can be seen via analytic Fredholm theory $([24])$ applied to

$$
(H-z)^{-1}=\left(H_{0}-z\right)^{-1}\left(1+V\left(H_{0}-z\right)^{-1}\right)^{-1},
$$

because

- $F(z):=V\left(H_{0}-z\right)^{-1}$ is an analytic function of $z \in \mathbb{C} \backslash[0, \infty)$ with values in the space of compact operators, and

- $(1-F(z))^{-1}$ exists for $z \in \mathbb{C} \backslash[0, \infty)$ with sufficiently large $|\Im z|$.

Since $H$ is non-self-adjoint, $m_{\lambda}$ is in general different from the geometric multiplicity defined by

$$
g_{\lambda}:=\operatorname{dim}\left\{u \in L^{2}\left(\mathbb{R}^{d}\right):(H-\lambda) u=0\right\},
$$

and we count the number of eigenvalues according to their algebraic multiplicities.

Then, the following estimates for the sum of moments of eigenvalues of $H$ outside the cone $\mathcal{C}_{\kappa}:=\{z \in \mathbb{C}:|\Im z|<\kappa \Re z\}$ for any positive constant $\kappa$ are proven:

Theorem 1.1 (Frank, Laptev, Lieb and Seiringer; [12]). Let $d \geq 1$ and $\gamma \geq 1$. Suppose that $V \in L^{\gamma+d / 2}\left(\mathbb{R}^{d} ; \mathbb{C}\right)$. Then, for any $\kappa>0$,

$$
\sum_{\sigma_{\mathrm{d}}(H) \cap \mathcal{C}_{\kappa}^{\mathrm{c}}}|\lambda|^{\gamma} \leq C_{\gamma, d}\left(1+\frac{2}{\kappa}\right)^{\gamma+d / 2} \int_{\mathbb{R}^{d}}|V(x)|^{\gamma+d / 2} \mathrm{~d} x
$$

where $\mathcal{C}_{\kappa}^{\mathrm{c}}$ is the complement set of $\mathcal{C}_{\kappa}$ and

$$
C_{\gamma, d}:=2^{1+\gamma / 2+d / 4} L_{\gamma, d}
$$


and $L_{\gamma, d}$ is the constant of (1.3). In particular,

$$
\sum_{\sigma_{\mathrm{d}}(H) \cap\{\Re z \leq 0\}}|\lambda|^{\gamma} \leq C_{\gamma, d} \int_{\mathbb{R}^{d}}|V(x)|^{\gamma+d / 2} \mathrm{~d} x .
$$

Frank et al.([12]) conjecture that (1.7) and (1.9) in Theorem 1.1 hold for $\gamma$ satisfying (1.4). On the other hand, observing that (1.3) is equivalent to

$$
\sum_{n=1}^{\infty} \frac{\operatorname{dist}\left(\lambda_{n} ;[0, \infty)\right)^{\gamma+d / 2}}{\left|\lambda_{n}\right|^{d / 2}} \leq L_{\gamma, d} \int_{\mathbb{R}^{d}} V_{-}(x)^{\gamma+d / 2} \mathrm{~d} x
$$

where $\operatorname{dist}(x ; \Omega)$ is a distance from a point $x$ to the domain $\Omega$ and $\left\{\lambda_{n}\right\}$ are negative eigenvalues of $H$ given by (1.2) , Demuth, Hansmann and Katriel([6]) proposed to study the estimate:

$$
\sum_{\lambda \in \sigma_{\mathrm{d}}(H)} \frac{\operatorname{dist}(\lambda ;[0, \infty))^{\gamma+d / 2}}{|\lambda|^{d / 2}} \leq C_{\gamma, d} \int_{\mathbb{R}^{d}}|V(x)|^{\gamma+d / 2} \mathrm{~d} x
$$

for the constant $C_{\gamma, d}$ independent of $V \in L^{\gamma+d / 2}\left(\mathbb{R}^{d} ; \mathbb{C}\right)$ and for $\gamma$ and $d$ satisfying (1.4). They ([8]) actually proved by applying (1.7) that for any $\gamma \geq 1$ and $0<\tau<1$,

$$
\sum_{\lambda \in \sigma_{\mathrm{d}}(H)} \frac{\operatorname{dist}(\lambda ;[0, \infty))^{\gamma+d / 2+\tau}}{|\lambda|^{d / 2+\tau}} \leq C_{\gamma, d, \tau} \int_{\mathbb{R}^{d}}|V(x)|^{\gamma+d / 2} \mathrm{~d} x
$$

with constant

$$
C_{\gamma, d, \tau}=\frac{\text { const. }}{\tau} \text {. }
$$

Note that this constant blows up as $\tau \downarrow 0$ (see also [7] where a similar estimate is obtained).

Related to this problem, Frank and Sabin([14]) proved that if $d \geq 1$ and $V \in L^{p}\left(\mathbb{R}^{d} ; \mathbb{C}\right)$ such that

$$
\begin{cases}p=1, & d=1, \\ 1<p \leq \frac{3}{2}, & d=2, \\ \frac{d}{2}<p \leq \frac{d+1}{2}, & d \geq 3,\end{cases}
$$

then

$$
\sum_{\lambda \in \sigma_{\mathrm{d}}(H)} \frac{\operatorname{dist}(\lambda ;[0, \infty))}{|\lambda|^{(1-\varepsilon) / 2}} \leq C_{p, d, \varepsilon}\left(\int_{\mathbb{R}^{d}}|V(x)|^{p} \mathrm{~d} x\right)^{(1+\varepsilon) /(2 p-d)}
$$


where $\varepsilon$ is the non-negative number fulfilling:

$$
\left.\begin{array}{ll}
\varepsilon>1, & d=1 \\
\varepsilon \geq 0, & d \geq 2 \text { and } \frac{d}{2} \leq p \leq \frac{d^{2}}{2 d-1} \\
\varepsilon>\frac{(2 d-1) p-d^{2}}{d-p}, & d \geq 2 \text { and } \frac{d^{2}}{2 d-1} \leq p \leq \frac{d+1}{2}
\end{array}\right\} .
$$

In recent years, Cuenin, Laptev, Safronov etc. studied the eigenvalues of $H$ which are close to $[0, \infty)$. For example, they $([22)$ proved that if $\Re V \geq 0$ is bounded and $\Im V \in L^{p}\left(\mathbb{R}^{d}\right)$ and

$$
\left.\begin{array}{l}
p \geq 1, \quad d=1 \\
p>\frac{d}{2}, \quad d \geq 2
\end{array}\right\}
$$

then one has

$$
\sum_{\lambda \in \sigma_{\mathrm{d}}(H)}\left(\frac{\Im \lambda}{|\lambda+1|^{2}+1}\right)_{+}^{p} \leq C_{p, d} \int_{\mathbb{R}^{d}}(\Im V)_{+}(x)^{p} \mathrm{~d} x
$$

where

$$
C_{p, d}:=(2 \pi)^{-d} \int_{\mathbb{R}^{d}} \frac{\mathrm{d} \xi}{\left(|\xi|^{2}+1\right)^{p}}
$$

\section{Assumption and Main Results}

We write $(\cdot, \cdot)$ for the $L^{2}\left(\mathbb{R}^{d}\right)$-inner product and $\|\cdot\|$ for the $L^{2}\left(\mathbb{R}^{d}\right)$-norm:

$$
(f, g):=\int_{\mathbb{R}^{d}} f(x) \overline{g(x)} \mathrm{d} x, \quad\|f\|:=(f, f)^{1 / 2} .
$$

\subsection{Dilation Analytic Method for Complex Potentials}

We consider the 1-parameter unitary group $U(\theta), \theta \in \mathbb{R}$, on $L^{2}\left(\mathbb{R}^{d}\right)$ defined by

$$
U(\theta) u(x):=\mathrm{e}^{d \theta / 2} u\left(\mathrm{e}^{\theta} x\right), \quad u \in L^{2}\left(\mathbb{R}^{d}\right) .
$$

We now suppose that $V$ fulfills the following assumption. Recall that $\mathcal{D}(T)$ is the domain of the operator $T$. Moreover, we write $\mathbf{B}\left(S_{1}, S_{2}\right)$ for the space of bounded operators from the space $S_{1}$ to the space $S_{2}$, in particular $\mathbf{B}(S)$ is $\mathbf{B}(S, S)$ if $S_{1}=S_{2}=S$. 
Assumption 2.1. Let $d, \gamma \geq 1$. We assume the followings from beginning to end.

a) $V$ is the multiplication operator with the complex-valued measurable function satisfying $V \in L^{\gamma+d / 2}\left(\mathbb{R}^{d} ; \mathbb{C}\right)$.

b) The operator $V$ is $H_{0}$-compact, that is, $\mathcal{D}(V) \supset \mathcal{D}\left(H_{0}\right)=H^{2}\left(\mathbb{R}^{d}\right)$ and $V\left(H_{0}+1\right)^{-1}$ is compact.

c) The function $V_{\theta}(x):=V\left(\mathrm{e}^{\theta} x\right)$ originally defined for $\theta \in \mathbb{R}$ has an analytic continuation into the complex strip

$$
\mathscr{S}_{\alpha}:=\{z \in \mathbb{C}:|\Im z|<\alpha\} \text { for some } \alpha>0
$$

as an $L^{\gamma+d / 2}\left(\mathbb{R}^{d} ; \mathbb{C}\right)$-valued function.

d) The function $V_{\theta}\left(H_{0}+1\right)^{-1}$ originally defined for $\theta \in \mathbb{R}$ can be extended to $\mathscr{S}_{\alpha}$ as a $\mathbf{B}\left(L^{2}\left(\mathbb{R}^{d}\right)\right)$-valued analytic function.

In what follows, we fix $d, \gamma \geq 1$. We call $V$ fulfilling Assumption 2.1 the dilation analytic complex potential on $\mathscr{S}_{\alpha}$. We define

$$
\left\{\begin{array}{l}
H_{0}(\theta):=\mathrm{e}^{-2 \theta} H_{0}, \\
H(\theta):=U(\theta) H U(\theta)^{-1}=H_{0}(\theta)+V_{\theta}=\mathrm{e}^{-2 \theta}\left(H_{0}+\mathrm{e}^{2 \theta} V_{\theta}\right)
\end{array}\right.
$$

for $\theta \in \mathscr{S}_{\alpha}$. It is obvious that $H_{0}(\theta)$ and $H(\theta)$ are operator-valued holomorphic functions of type (A) of $\theta \in \mathscr{S}_{\alpha}$ in the sense of Kato ([16]). Moreover

$$
H\left(\theta+\theta^{\prime}\right)=U\left(\theta^{\prime}\right) H(\theta) U\left(\theta^{\prime}\right)^{-1} ; \quad \theta \in \mathscr{S}_{\alpha}, \theta^{\prime} \in \mathbb{R},
$$

since this is true for $\theta \in \mathbb{R}$ and both sides of (2.3) are $\mathbf{B}\left(H^{2}\left(\mathbb{R}^{d}\right) ; L^{2}\left(\mathbb{R}^{d}\right)\right)$ valued analytic functions of $\theta \in \mathscr{S}_{\alpha}$. In particular, $\sigma(H(\theta))$ is independent of $\Re \theta$.

The following result for real-valued potentials is a well known fact. We pay attention to that the same result holds for complex-valued potentials.

Proposition 2.1. If $V$ is a dilation analytic complex potential on $\mathscr{S}_{\alpha}$, one has

$$
\sigma_{\text {ess }}(H(\theta))=\mathrm{e}^{-2 \theta}[0, \infty)=\left\{\mathrm{e}^{-2 \theta} x: x \in[0, \infty)\right\}
$$

for any $\theta \in \mathscr{S}_{\alpha}$. 
Proof. Define, for fixed $\theta \in \mathscr{S}_{\alpha}$,

$$
\tilde{H}(\theta):=H_{0}+\mathrm{e}^{2 \theta} V_{\theta}=\mathrm{e}^{2 \theta} H(\theta) .
$$

That $\sigma(\tilde{H}(\theta)) \backslash[0, \infty)$ consists of isolated eigenvalues of finite multliplicities may be proved as previously by the argument using analytic Fredholm theory for

$$
F(z):=-\mathrm{e}^{2 \theta} V_{\theta}\left(H_{0}-z\right)^{-1} .
$$

In particular, $\sigma_{\text {ess }}(\tilde{H}(\theta)) \subset[0, \infty)$. We show that $[0, \infty) \subset \sigma_{\text {ess }}(\tilde{H}(\theta))$. Suppose that there is an open interval $(a, b) \subset[0, \infty)$ such that

$$
(a, b) \subset \mathbb{C} \backslash \sigma(\tilde{H}(\theta)) .
$$

Then, $\sigma\left(H_{0}\right) \cap(a, b)$ must be a discrete set by virtue of the argument above where the roles of $\tilde{H}(\theta)$ and $H_{0}$ are replaced. This is of course impossible and $[0, \infty) \subset \sigma_{\text {ess }}(\tilde{H}(\theta))$. Therefore,

$$
\sigma_{\mathrm{ess}}(\tilde{H}(\theta))=\sigma_{\mathrm{ess}}\left(H_{0}\right)=[0, \infty),
$$

and

$$
\sigma_{\text {ess }}(H(\theta))=\mathrm{e}^{-2 \theta} \sigma_{\text {ess }}(\tilde{H}(\theta))=\mathrm{e}^{-2 \theta}[0, \infty)
$$

by (2.5) and (2.6).

Remark 2.1. (2.4) is immediately derived by Weyl's theorem for essential spectrums if $H$ is self-adjoint. (See e.g. Theorem XIII.36 of [25].)

\subsection{Estimates on Eigenvalues in $\mathbb{C} \backslash[0, \infty)$}

The main result in the present paper is the following theorem.

Theorem 2.1. Let $V$ be a dilation analytic complex potential on $\mathscr{S}_{\alpha}$ with $\alpha>\pi / 4$. Suppose that $C_{\gamma, d}$ is the constant given by (1.8). Then, one has

$$
\sum_{\lambda \in \sigma_{\mathrm{d}}(H) \cap\left[\mathbb{C}^{ \pm} \cup(-\infty, 0)\right]}|\lambda|^{\gamma} \leq C_{\gamma, d} \int_{\mathbb{R}^{d}}\left|V_{ \pm \pi \mathrm{i} / 4}(x)\right|^{\gamma+d / 2} \mathrm{~d} x .
$$

As follows, Theorem 2.1 derives the Lieb-Thirring type inequality for all isolated eigenvalues of the non-self-adjoint Schrödinger operator with the dilation analytic complex potential. 
Corollary 2.1. If $V$ is a dilation analytic complex potential on $\mathscr{S}_{\alpha}$ with $\alpha>\pi / 4$, then one has

$$
\sum_{\lambda \in \sigma_{\mathrm{d}}(H)}|\lambda|^{\gamma} \leq C_{\gamma, d} \int_{\mathbb{R}^{d}}\left(\left|V_{\pi \mathrm{i} / 4}(x)\right|^{\gamma+d / 2}+\left|V_{-\pi \mathrm{i} / 4}(x)\right|^{\gamma+d / 2}\right) \mathrm{d} x
$$

where $C_{\gamma, d}$ is the constant of (1.8).

Remark 2.2. It is predicted that in general the integrals on the right-hand side of (2.7) are bigger than $\int|V(x)|^{\gamma+d / 2} \mathrm{~d} x$. (See the following example.) However, we can of course take minimum constants $C^{ \pm}$satisfying

$$
\int_{\mathbb{R}^{d}}\left|V_{ \pm \pi \mathrm{i} / 4}(x)\right|^{\gamma+d / 2} \mathrm{~d} x \leq C^{ \pm} \int_{\mathbb{R}^{d}}|V(x)|^{\gamma+d / 2} \mathrm{~d} x
$$

respectively, so we come to the conclusion that the Lieb-Thirring inequality (with a worse constant) for all isolated eigenvalues of $\mathrm{H}$ with the dilation analytic complex potential establishes from Corollary 2.1.

$$
\sum_{\lambda \in \sigma_{\mathrm{d}}(H)}|\lambda|^{\gamma} \leq C \int_{\mathbb{R}^{d}}|V(x)|^{\gamma+d / 2} \mathrm{~d} x, \quad C:=2 \max \left\{C_{\gamma, d} C^{-}, C_{\gamma, d} C^{+}\right\} .
$$

Here, in general $C^{ \pm}$probably depend on $V$ in both cases.

Example. We consider a 1-dimensional complex potential

$$
V(x)=\frac{c}{\left(1+x^{2}\right)^{s}}, \quad x \in \mathbb{R}
$$

where $c \in \mathbb{C}$ and $1 / 2<s<1$. $V$ is a dilation analytic complex potential on $\mathscr{S}_{\alpha}$ with $\alpha \in(\pi / 4, \pi / 2)$. In fact, a singularity appears if $\theta=\pi \mathrm{i} / 2$ since

$$
V_{\theta}(x)=\frac{c}{\left(1+\mathrm{e}^{2 \theta} x^{2}\right)^{s}},
$$

moreover $V_{\theta} \in L^{\gamma+1 / 2}\left(\mathbb{R}^{d}\right)$ if and only if

$$
\frac{1}{2 \gamma+1}<s<\frac{2}{2 \gamma+1} \text {. }
$$

In particular $\gamma=1$, we obtain

$$
\begin{aligned}
\int_{-\infty}^{\infty}\left|V_{ \pm \pi \mathrm{i} / 4}(x)\right|^{3 / 2} \mathrm{~d} x & =\int_{-\infty}^{\infty} \frac{|c|^{3 / 2}}{{\sqrt{1+x^{4}}}^{3 s / 2}} \mathrm{~d} x \\
& \geq \int_{-\infty}^{\infty} \frac{|c|^{3 / 2}}{\left(1+x^{2}\right)^{3 s / 2}} \mathrm{~d} x \\
& =\int_{-\infty}^{\infty}|V(x)|^{3 / 2} \mathrm{~d} x
\end{aligned}
$$


as expected. By the way, if $V: \mathbb{R}^{d} \rightarrow \mathbb{C}$ is a complex-valued potential like (2.8), that is, if $V$ satisfies that

$$
|V(x)| \leq \frac{\text { const. }}{\left(1+|x|^{2}\right)^{s}}, \quad \frac{1}{2}<s<1,
$$

it is known([26]) that all non-real eigenvalues of the non-self-adjoint Schrödinger operator $H=-\Delta+V$ are in a disc of a finite radius.

We denote the number of the isolated eigenvalues of the operator $T$ in a domain $\Omega$ by $N(T ; \Omega)$. If we add another condition besides Assumption 2.1 as follows, we can mention the number of all isolated eigenvalues of $H$.

Corollary 2.2. Let $H$ be a dissipative Schrödinger operator with the dilation analytic complex potential on $\mathscr{S}_{\alpha}$ with $\alpha>\pi / 4$. That is, we suppose that

i) the function $\mathbb{R}^{d} \ni x \mapsto V(x)$ satisfies $\Im V(x)<0$ for all $x \in \mathbb{R}^{d}$ and

ii) there exists some constant $C$ such that

$$
|\Re V(x)|,|\Im V(x)| \leq C\langle x\rangle^{-\rho}
$$

outside the sufficiently large sphere, where $\langle x\rangle:=\left(1+|x|^{2}\right)^{1 / 2}$ and $\rho>1$.

Then, one has

$$
N(H ; \mathbb{C} \backslash[0, \infty)) \leq \tilde{C}_{\gamma, d} \int_{\mathbb{R}^{d}}\left(\left|V_{\pi \mathrm{i} / 4}(x)\right|^{\gamma+d / 2}+\left|V_{-\pi \mathrm{i} / 4}(x)\right|^{\gamma+d / 2}\right) \mathrm{d} x
$$

where $\tilde{C}_{\gamma, d}:=C_{\gamma, d} / \inf _{\lambda \in \sigma_{\mathrm{d}}(H)}|\lambda|^{\gamma}$.

Proof. We put $\Lambda:=\inf _{\lambda \in \sigma_{\mathrm{d}}(H)}|\lambda|^{\gamma}$ for simplicity. First,

$$
\sum_{\lambda \in \sigma_{\mathrm{d}}(H)}|\lambda|^{\gamma} \geq\left(\inf _{\lambda \in \sigma_{\mathrm{d}}(H)}|\lambda|^{\gamma}\right) \sum_{\lambda \in \sigma_{\mathrm{d}}(H)} 1=\Lambda N(H ; \mathbb{C} \backslash[0, \infty)) .
$$

Next, it is proved that 0 is not an accumulation point of isolated eigenvalues of $H$ if $H$ is dissipative and $V$ satisfies (2.9) (See Theorem 1.1 of [28]), so $\Lambda>0$. Hence, (2.10) holds from the above. 


\section{Proof of Theorem 2.1}

The estimate (1.9) of Theorem 1.1plays an important role in the proof of the main theorem. The points of a proof of Theorem 2.1 are that isolated eigenvalues of $H$ and their multiplicities are invariant under complex dilation. Let us prove them by dividing into two lemmmas.

We begin with the following lemma. Recall that $\sigma(H(\theta))$ is discrete in $\mathbb{C} \backslash \sigma_{\text {ess }}(H(\theta))$ and all $\lambda \in \sigma_{\mathrm{d}}(H(\theta))$ have finite algebraic multiplicities.

Lemma 3.1. Suppose that $0<\alpha<\pi / 2$ and $\theta \in \mathscr{S}_{\alpha} \cap \mathbb{C}^{ \pm}$. Then, for $\lambda \in \mathbb{C}^{ \pm}, \lambda \in \sigma_{\mathrm{d}}(H)$ if and only if $\lambda \in \sigma_{\mathrm{d}}(H(\theta))$.

Proof. We prove only the case $\theta \in \mathscr{S}_{\alpha} \cap \mathbb{C}^{+}$and $\lambda \in \mathbb{C}^{+}$. The other case may be proved simliarly. Suppose that $\lambda(\theta) \in \mathbb{C}^{+}$is an eigenvalue of $H(\theta)$ for $\theta \in \mathscr{S}_{\alpha} \cap \mathbb{C}^{+}$. We show that if $\lambda$ is an eigenvalue of $H\left(\theta_{0}\right)$ for $\theta_{0} \in \mathscr{S}_{\alpha} \cap \mathbb{C}^{+}$ then $\lambda$ is an eigenvalue of $H(\theta)$ for all $\theta \in \mathscr{S}_{\alpha} \cap \mathbb{C}^{+}$. To see this, we recall that $\{H(\theta)\}_{\theta \in \mathscr{S}_{\alpha} \cap \mathbb{C}^{+}}$is an analytic family of type (A) in the sense of Kato. Then, Theorem 1.8 of Chapter VII $\S 1.3$ (or Chapter II $\S 1$ ) of [16] implies that those $\lambda(\theta) \in \sigma_{\mathrm{d}}(H(\theta))$ such that $\lambda(\theta) \rightarrow \lambda$ as $\theta \rightarrow \theta_{0}$ are given by the branches of one or several analytic functions as Puiseux series. Moreover, as remarked above, $\lambda(\theta)$ is independent of $\Re \theta$. It follows that $\lambda(\theta)=\lambda$ for all $\theta$ near $\theta_{0}$, and $\lambda(\theta)=\lambda, \theta \in \mathscr{S}_{\alpha} \cap \mathbb{C}^{+}$.

Lemma 3.2. Let $V$ be a dilation analytic complex potential on $\mathscr{S}_{\alpha}$ with $\alpha>\pi / 4$. Suppose that $\lambda \in \mathbb{C}^{ \pm}$is an eigenvalue of $H$ and $\theta \in \mathscr{S}_{\alpha} \cap \mathbb{C}^{ \pm}$. Then, the algebraic multiplicity of $\lambda$ as eigenvalue of $H(\theta)$ coincides with that of $\lambda$ as eigenvalue of $H$.

Proof. We define two Riesz projections onto the generalized eigenspaces:

$$
P_{\lambda}:=\frac{-1}{2 \pi \mathrm{i}} \oint_{\Gamma_{\varepsilon}}(H-z)^{-1} \mathrm{~d} z, \quad P_{\lambda}(\theta):=\frac{-1}{2 \pi \mathrm{i}} \oint_{\Gamma_{\varepsilon}}(H(\theta)-z)^{-1} \mathrm{~d} z
$$

for $\theta \in \mathscr{S}_{\alpha} \cap \mathbb{C}^{ \pm}$, where $\Gamma_{\varepsilon}$ is the contour around $\lambda$ defined by

$$
\Gamma_{\varepsilon}:=\{z \in \mathbb{C}:|z-\lambda|=\varepsilon\}
$$

with sufficient small $\varepsilon>0$ such that $\Gamma_{\varepsilon}$ does not enclose any other point in $\sigma(H)$ or $\sigma(H(\theta))$ except $\lambda$. Here, notice that $P_{\lambda}$ is not necessary an orthogonal projection, since $H$ is non-self-adjoint. It is well known that $P_{\lambda}, P_{\lambda}(\theta)$ are projections and

$$
P_{\lambda}^{2}=P_{\lambda}, \quad P_{\lambda}(\theta)^{2}=P_{\lambda}(\theta)
$$


onto the respective generalized eigenspaces of $H$ and $H(\theta)$ associated with the eigenvalue $\lambda$. It follows that

$$
m_{\lambda}=\operatorname{rank} P_{\lambda}, \quad m_{\lambda(\theta)}=\operatorname{rank} P_{\lambda}(\theta) .
$$

We write $P_{\lambda}^{\prime}(\theta)$ for the orthogonal projection onto $M_{\lambda}(\theta):=P_{\lambda}(\theta) L^{2}\left(\mathbb{R}^{d}\right)$. In order to prove this lemma, we show that the dimension of $M_{\lambda}(\theta)$ is independent of $\theta \cdot P_{\lambda}(\theta)$ is analytic with respect to $\theta$. It is known that

$$
\left\|P_{\lambda}^{\prime}(\theta)-P_{\lambda}^{\prime}(\sigma)\right\| \leq\left\|P_{\lambda}(\theta)-P_{\lambda}(\sigma)\right\|
$$

for any $\theta, \sigma \in \mathscr{S}_{\alpha}$ (see Theorem 6.35 in Chapter I $\S 6.8$ of [16]). Since $P_{\lambda}(\theta) \rightarrow P_{\lambda}(\sigma)$ as $\theta \rightarrow \sigma$ in norm by the analyticity of $P_{\lambda}(\theta)$ (see (3.1)) and

$$
\left(P_{\lambda}^{\prime}(\theta)-P_{\lambda}^{\prime}(\sigma)\right)^{2}+\left(1-P_{\lambda}^{\prime}(\theta)-P_{\lambda}^{\prime}(\sigma)\right)^{2}=1
$$

one has

$$
\left\|P_{\lambda}^{\prime}(\theta)-P_{\lambda}^{\prime}(\sigma)\right\|<1 .
$$

Thus, $P_{\lambda}^{\prime}(\theta)$ and $P_{\lambda}^{\prime}(\sigma)$ are unitarily equivalent by Theorem 6.32 in Chapter I $\S 6.8$ of [16]. Hence

$$
\operatorname{dim} M_{\lambda}(\theta)=\operatorname{dim} M_{\lambda}(\sigma),
$$

so the proof of this lemma ends by putting $\sigma=0$.

We denote $\mathbb{R}_{ \pm}:=\{ \pm x: x>0\}$ and i $\mathbb{R}_{ \pm}:=\left\{\mathrm{i} x: x \in \mathbb{R}_{ \pm}\right\}$.

Proof of Theorem 2.1. For eigenvalues $\tilde{\lambda}(\theta)$ of $\tilde{H}(\theta)=\mathrm{e}^{2 \theta} H(\theta)$, we write

$$
\lambda(\theta)=\mathrm{e}^{-2 \theta} \tilde{\lambda}(\theta)
$$

for the corresponding eigenvalue of $H(\theta), \theta \in \mathscr{S}_{\alpha}$.

We first set $\theta=\pi \mathrm{i} / 4$. Lemma 3.1, Lemma 3.2 and (3.3) imply that

$$
\mathrm{e}^{\pi \mathrm{i} / 2}\left(\sigma_{\mathrm{d}}(H) \cap\left(\mathbb{C}^{+} \cup \mathbb{R}_{-}\right)\right)=\sigma_{\mathrm{d}}(\tilde{H}(\pi \mathrm{i} / 4)) \cap\left(\{\Re z<0\} \cup \mathrm{i} \mathbb{R}_{-}\right)
$$

including their multiplicities. We next set $\theta=-\pi \mathrm{i} / 4$. We likewise have

$$
\mathrm{e}^{-\pi \mathrm{i} / 2}\left(\sigma_{\mathrm{d}}(H) \cap\left(\mathbb{C}^{-} \cup \mathbb{R}_{-}\right)\right)=\sigma_{\mathrm{d}}(\tilde{H}(-\pi \mathrm{i} / 4)) \cap\left(\{\Re z<0\} \cup \mathrm{i} \mathbb{R}_{+}\right)
$$

including their multiplicities. We write $\mathfrak{S}_{\mp}(\tilde{H}( \pm \pi \mathrm{i} / 4))$ for the right-hand side of (3.4) and (3.5) respectively, and apply the estimate (1.9) of Theorem 2.1 to $\tilde{H}( \pm \pi \mathrm{i} / 4))=H_{0} \pm \mathrm{i} V_{\pi \mathrm{i} / 4}$. This implies

$$
\begin{aligned}
\sum_{\lambda \in \sigma_{\mathrm{d}}(H) \cap\left(\mathbb{C}^{ \pm} \cup \mathbb{R}_{-}\right)}|\lambda|^{\gamma} & =\sum_{\tilde{\lambda}( \pm \pi \mathrm{i} / 4) \in \mathfrak{G}_{\mp}(\tilde{H}( \pm \pi \mathrm{i} / 4))}|\tilde{\lambda}( \pm \pi \mathrm{i} / 4)|^{\gamma} \\
& \leq C_{\gamma, d} \int_{\mathbb{R}^{d}}\left|V_{ \pm \pi \mathrm{i} / 4}(x)\right|^{\gamma+d / 2} \mathrm{~d} x .
\end{aligned}
$$


Since $m_{\tilde{\lambda}(\pi \mathrm{i} / 4)}=m_{\tilde{\lambda}(-\pi \mathrm{i} / 4)}=m_{\lambda}$ for any $\lambda \in \sigma_{\mathrm{d}}(H) \cap\left(\mathbb{C}^{ \pm} \cup \mathbb{R}_{-}\right)$by virtue of Lemma 3.2, this completes the proof.

\section{ACKNOWLEDGEMENT}

The author heartily thank professor Kenji Yajima for advice and instruction. Moreover, the author would like to thank the referees of ROMP for precious advice and Dr. Ryszard Mrugała for guidance respectively.

\section{References}

[1] A. A. Abramov, A. Aslanyan and E. B. Davies, Bounds on Complex Eigenvalues and Resonances, Journal of Physics A 34 (2001), No.1, 57-72

[2] J. Aguilar and J. M. Combes, A Class of Analytic Perturbations for One-body Schrödinger Hamiltonians, Commu. Math. Phys. 22 (1971), 269-279

[3] M. Aizenman and E. H. Lieb, On semi-classical bounds for eigenvalues of Schrödinger operators, Physics Letters Vol.66 A, No.6 (1978), 427-429

[4] J. - C. Cuenin, A. Laptev and C. Tretter, Eigenvalue Estimates for Non-Selfadjoint Dirac Operators on the Real Line, Ann. Henri Poincaré 15 (2014), 707-736

[5] H. L. Cycon, R. G. Froese, W. Kirsch and B. Simon, Schrödinger Operators with Applications to Quantum Mechanics and Global Geometry, Springer (2008)

[6] M. Demuth, M. Hansmann and G. Katriel, Lieb-Thirring Type Inequalities for Schödinger Operators with a Complex-Valued Potential, Integral Equations and Operator Theory 75 (2013), 1-5

[7] M. Demuth, M. Hansmann and G. Katriel, Eigenvalues of Non-self-adjoint Operators: A Comparison of Two Approaches, Operator Theory: Advances and Applications, Vol.232 (2013), 107-163

[8] M. Demuth, M. Hansmann and G. Katriel, On the Discrete Spectrum of Nonself-adjoint Operators, Journal of Functional Analysis 257 (2009), 2742-2759

[9] J. Dolbeault, A. Laptev and M. Loss, Lieb-Thirring inequalities with improved constants, J. Eur. Math. Soc. 10 (2008), 1121-1126

[10] R. L. Frank, Eigenvalue Bounds for Schrödinger Operators with Complex Potentials, Bull. London Math. Soc. 43 (2011), 745-750

[11] R. L. Frank, D. Hundertmark, M. Jex and P. T. Nam, The Lieb-Thirring inequality revisited, arXiv:1808.09017v1 [math-ph], 27 Aug. 2018 
[12] R. L. Frank, A. Laptev, E. H. Lieb and R. Seiringer, Lieb-Thirring Inequalities for Schrödinger Operators with Complex-valued Potentials, Letters in Mathematical Physics 77 (2006), 309-316

[13] R. L. Frank, A. Laptev and O. Safronov, On the number of eigenvalues of Schrödinger operators with complex potentials, Journal of the London Mathematical Society, Volume 94, Issue 2 (2016), 377-390

[14] R. L. Frank and J. Sabin, Restriction Theorems for Orthonormal Functions, Strichartz Inequalities, and Uniform Sobolev Estimates, arXiv:1404.2817v3 [math-ph], 27 May 2014

[15] B. Helffer and D. Robert, Riesz means of bounded states and semi-classical limit connected with a Lieb-Thirring conjecture II, Ann. Inst. H. Poincaré Phys. Théor. 53, No.2 (1990), 139-147

[16] T. Kato, Perturbation Theory for Linear Operators, Springer (1976)

[17] A. Laptev and O. Safronov, Eigenvalue Estimates for Schrödinger Operators with Complex Potentials, Commun. Math. Phys. 292 (2009), 29-54

[18] A. Laptev and T. Weidl, Recent Results on Lieb-Thirring inequalities, Journées "Équations aux Dérivées Partielles"(La Chapelle sur Erdre, 2000), Exp. No.XX,14, Univ. Nantes, Nantes, 2000

[19] A. Laptev and T. Weidl, Sharp Lieb-Thirring inequalities in high dimensions, Acta Math., 184 (2000), 87-111

[20] E. H. Lieb, Lieb-Thirring Inequalities, arXiv:math-ph/0003039v1, 27 Mar. 2000

[21] E. H. Lieb and M. Loss, Analysis (Second Edition), American Mathematical Society (2010)

[22] E. H. Lieb and R. Seiringer, The Stability of Matter in Quantum Mechanics, Cambridge University Press (2010)

[23] E. H. Lieb and W. Thirring, Inequalities for the Moments of the Eigenvalues of the Schrödinger Hamiltonian and Their Relation to Sobolev inequalities, With W. Thirring in Studies in Mathematical Physics, E. Lieb, B. Simon, A. Wightman eds. Inequalities, Springer-Verlag Berlin Heidelberg (2002)

[24] M. Reed and B. Simon, Methods of Modern Mathematical Physics I: Functional Analysis, Academic Press (1980)

[25] M. Reed and B. Simon, Methods of Modern Mathematical Physics IV: Analysis of Operators, Academic Press (1978)

[26] O. Safronov, Estimates for eigenvalues of the Schrödinger operator with a complex potential, Bull. London Math. Soc. 42 (2010), 452-456

[27] P. Šeba, The Complex Scaling Method for Dirac Resonances, Letters in Mathematical Physics 16 (1988), 51-59 
[28] X. P. Wang, Number of Eigenvalues for A Class of Non-self-adjoint Schrödinger Operators, J. Math. Pure Appl., 96(5) (2011), 409-422

[29] K. Yajima, Schrödinger Houteishiki I, Asakura-suugaku-taikei 5, Asakurashoten (2014) [Japanese] 